\title{
A New Method for Detecting the Quality of Special Road in Automobile Proving Ground
}

\author{
Huawen Yan, Dong Wang and Weigong Zhang a \\ School of Instrument Science and Engineering, Southeast University, Nanjing 210096, China \\ a zhangwg@seu.edu.cn
}

Keywords: wheel force transducer, proving ground, rain-flow counting method, special road.

\begin{abstract}
Automobile Proving Ground is the test platform for commercial vehicle manufacturers to test prototype vehicle in which the special roads can save test time and accelerate the speed of development of a new car. However, the pavement of the roads will inevitably be damaged with the extension of the service life, so accurate detection of the roads quality becomes very important. Traditionally, equipment similar to laser cross-section was used to detect pavement quality, the principle of which was to reflect pavement quality by detecting road surface roughness. This method ignores the elastic deformation of the roads itself when the vehicle is traveling on it and hardly compensate for the amendment. Therefore, this paper proposes a new method, which uses sensors that can collect road dynamic loads, to solve this problem. Through the analysis of the dynamic loads of the special roads, it not only completely solves the problem that the deformation of the road hardly be detected, but also quantify the characteristics of the road loads, and making the quality assessment of the special road more straightforward and more accurate..
\end{abstract}

\section{Introduction}

The prototype test is a key part to develop a new car, which is responsible for detecting the presence of defects in the previous part, and test out some of the important performance of the car, such as power, reliability, ride comfort, handling and durability and so on. Mass production is possible unless the prototype test has achieved a complete success. The special roads test in the automobile proving ground is an important part of the prototype test, which including cobblestone road test, wash plate road test, gravel road test, wave road test, Belgian road test, twisted road test, pit road test, stone road test, muddy road test and so on[1], some of the special roads are shown in Fig.1.
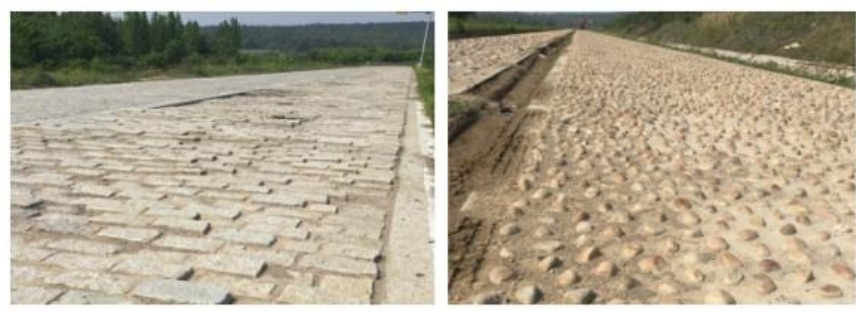
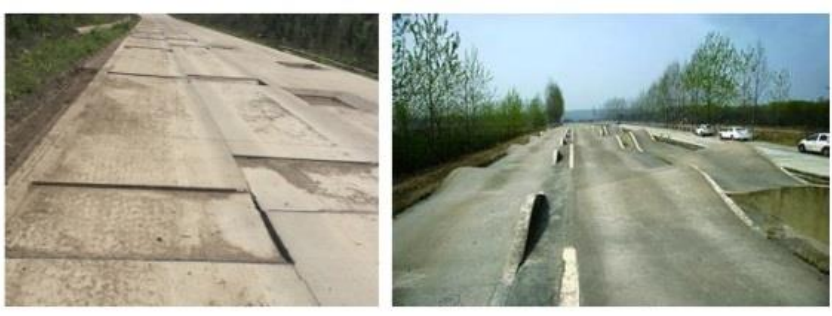

Fig.1: The special roads

Different special road gives the vehicle different frequency and different strength loads, and focusing on different aspects of performance testing. As the increase in road service time, the roads inevitably will be damaged. Traditionally, equipment such as laser cross-section was used to evaluate pavement quality [2]. Its core was evaluated by pavement roughness. The method assessing the road grade by road traffic department ignores the road and vehicle interaction process, because the road itself will produce elastic deformation while the vehicles pass the road, and the deformation affect the accuracy of the roughness. We know that the purpose of the special roads is to provide sufficient loads to speed up the testing of the vehicle, which is essentially different from the quality of the rated grade road, and thus there are inherent limitations in the assessment of the special road [3]. Therefore, this paper presents a new evaluation method based on wheel force transducer (WFT), which can accurately obtain the loads applied to the road by the vehicle, and then evaluate the road quality by the statistical 
analysis of the loads. This approach takes the interaction between the vehicle and the road into account, which is more comprehensive than the method of evaluating the road's geometric dimensions alone. The WFT is mounted on the axle and rotates with the wheel to dynamically measure the load applied to the road by the vehicle, and the multi-dimensional force sensor measures the force of the wheel in the three-dimensional direction.

\section{Collection of Special Road Load}

While a vehicle is on the road, because of the effect of the ground, some forces are applied to the wheel, namely the longitudinal force Fx, the lateral force Fy, the vertical force Fz [4], as shown in Fig. 2A. It is generally known that the role of force is mutual, so the wheel force is equivalent to the road load. The load can be collected by the WFT system, and the block diagram is shown in Fig. 2B. The hardware components of the system include elastomer, collection module, encoder, transmission module, central collection box, GPS module and laptop.
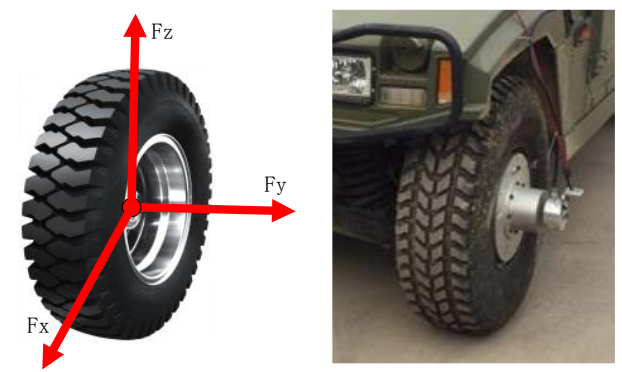

Fig. 2A: Forces diagram

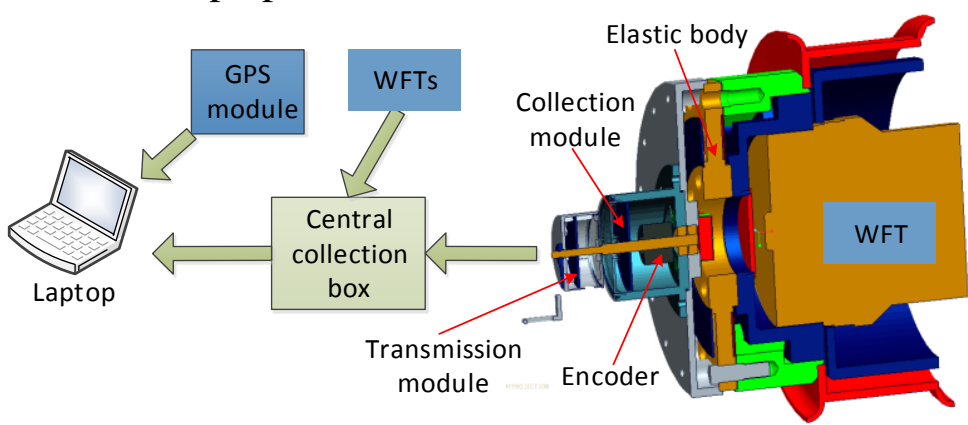

Fig. 2B: System block diagram

The WFT system is a vehicle-to-road coupling system, so the measured road load is related to multiple factors, which include road type, vehicle type, speed, tire pressure and so on. Road load of different road is not the same, which is the reason for setting various special roads. Different vehicles have different suspension types and weights, and their loads on the road are naturally different. In addition, the impact of speed and tire pressure on road loads is also evident. The vehicle used in this experiment is the military active off-road vehicle, which total weight is equal to curb weight plus weight of testers and equipment for the $3.7 \mathrm{t}$, and the tire pressure was adjusted to $2.7 \mathrm{Kg} / \mathrm{cm} 2$, the vehicle with WFT system as shown in Fig. 3.

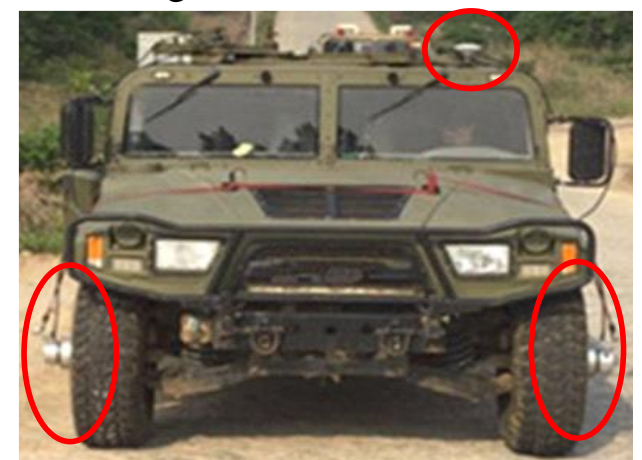

Fig. 3: The vehicle with WFT system

The sensors in the WFT system are mounted on the left and right wheels of the front axle. The special road used in the experiment was the cobblestone road, which includes a standard road and an old road. The speed of the vehicle is maintained at $40 \mathrm{~km} / \mathrm{h}$. The original loads data collected by the left WFT on the two roads are shown in Fig. 4 to Fig. 6. Owning to the right WFT is similar to the left one, so the original load curve of the left WFT is drawn alone for a brief description. 


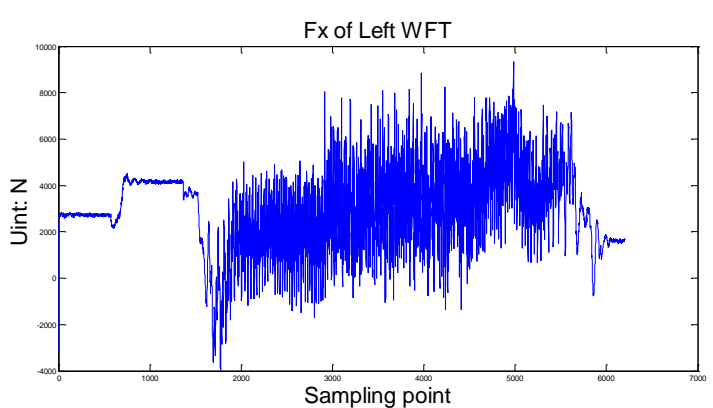

A: Fx .

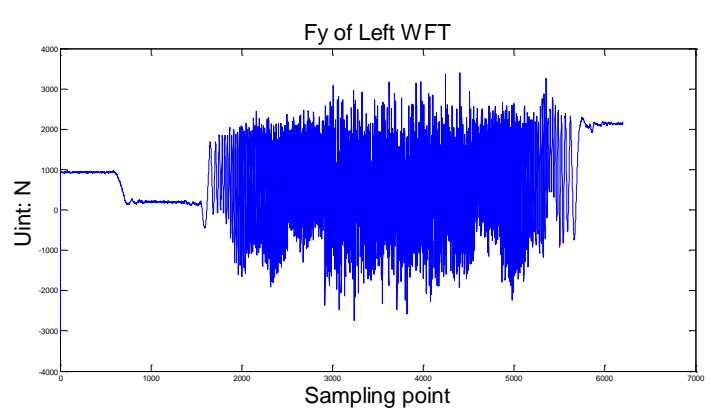

B: Fy .

Fig. 4: The original loads on the standard road

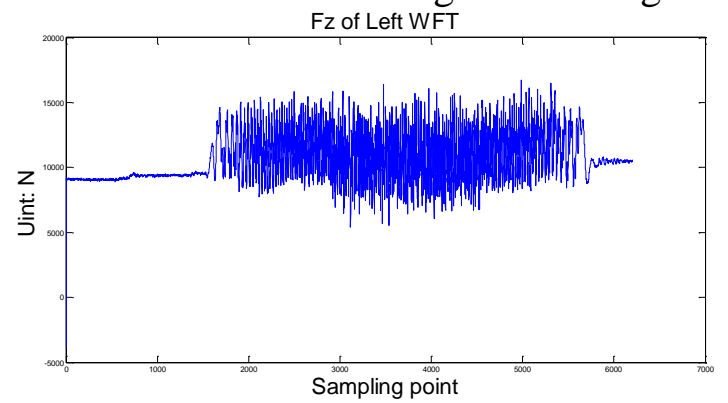

C: On the standard road

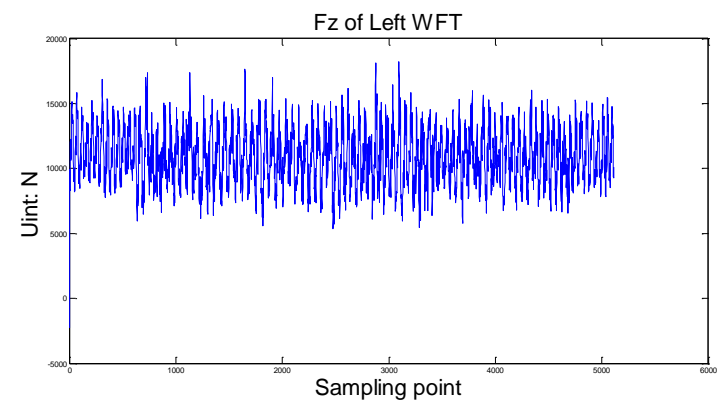

D: On the old road

Fig. 5: Fz

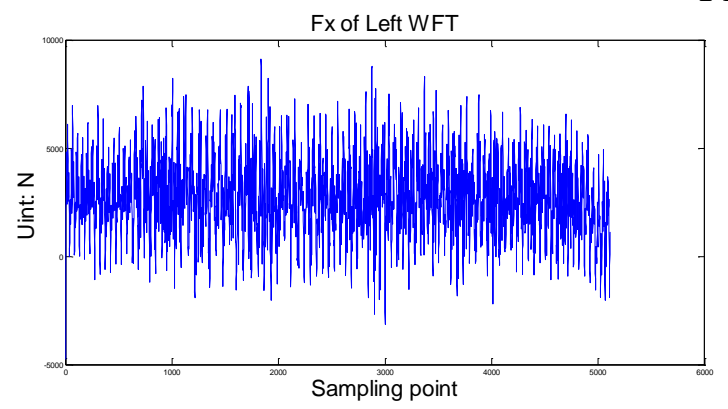

$\mathrm{E}: \mathrm{Fx}$.

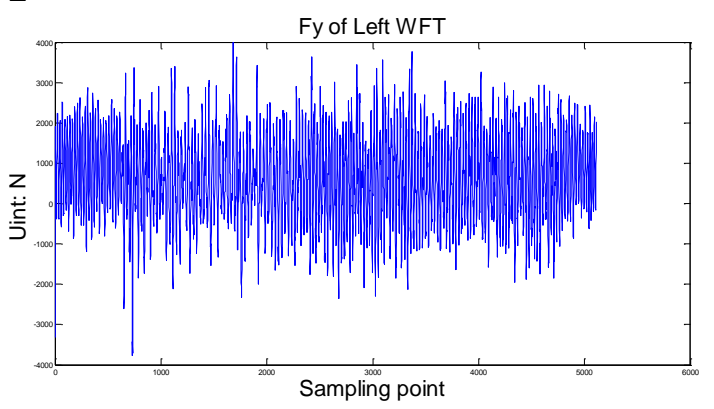

F: Fy .

Fig. 6: The original loads on the old road

Though the difference of original loads between the standard road and the old road is easy easily distinguishable from the curve form, but it is difficult to quantify the damage degree of the old road.

\section{Analysis of Special Road load}

In order to cope with the quantification problem, a widely used in the fatigue life calculation rain-flow counting method is introduced. The process of counting the load by rain-flow counting method reflects the memory characteristics of the material and has a clear mechanical concept, so the method has been widely used [5]. The basic counting rules are as follows:

1) The rain flows down the slope from the inside of the peak (valley) value of the load - time series;

2) A: Stop flowing when the absolute value of peak (valley) value is greater than the one of the initial peak (valley) value;

B: Stop flowing when encountering the above-mentioned rain;

3) Removing all the full cycles and writing down the valley and peak values for each cycle;

4) After the first stage of the counting will leave a divergent convergence load-time series, and converting it into a convergent-divergence of the load-time series, and then starting the second stage of the counting until the all of loads are counted. 


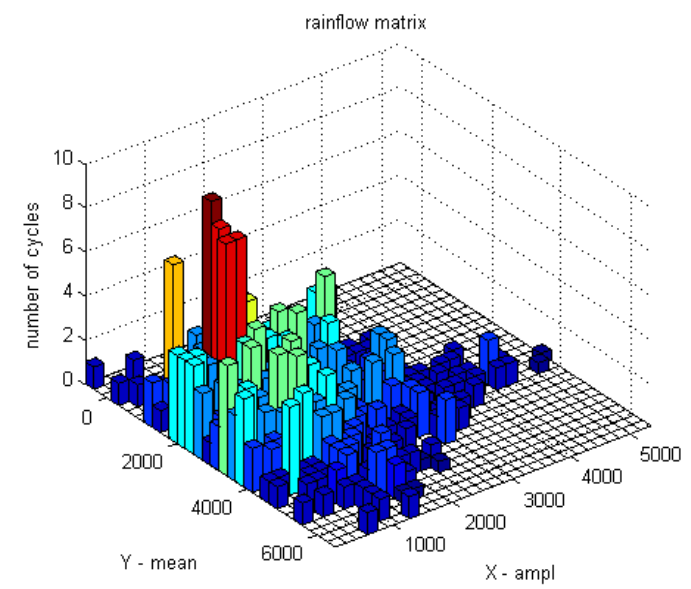

A: Rain-flow matrix of Fx

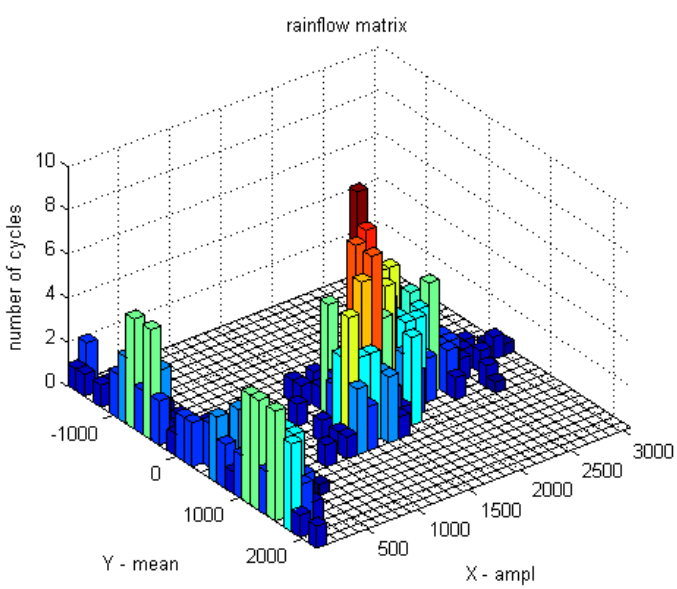

B: Rain-flow matrix of Fy

Fig. 7: Rain-flow matrix of load on the standard road.

It is easy to know that the rain flow counting method is very suitable for computer programming from the above counting rules. The original load data collected by the left WFT is input to the rain-flow counting program, and results of the rain-flow matrix is shown in Fig. 7 to Fig. 9.

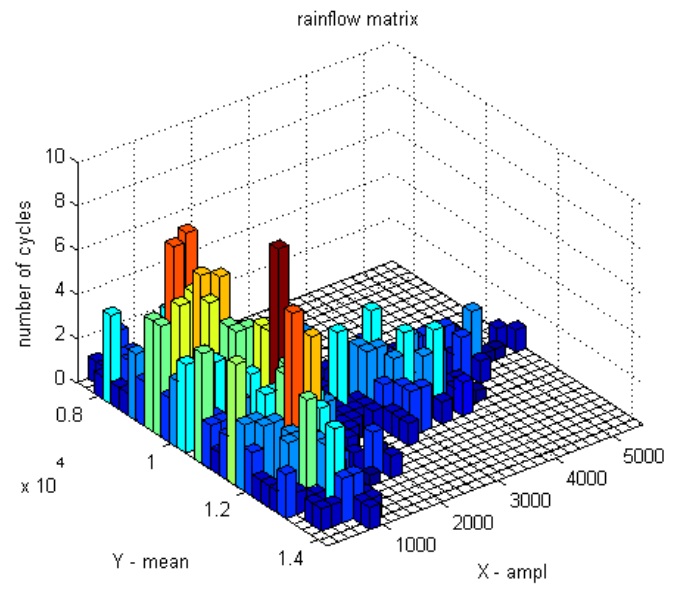

A: On the standard road.

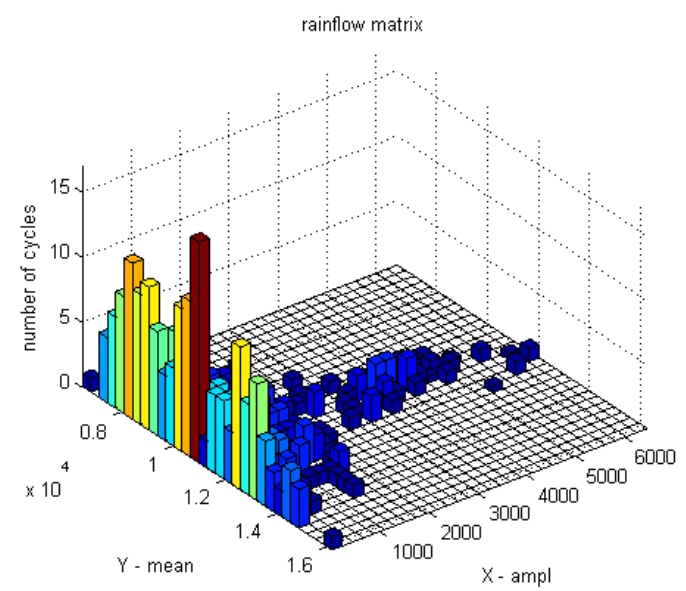

B: On the old road.

Fig. 8: Rain-flow matrix of Fz

The analysis of the rain-flow matrix of the right WFT is similar to the left, which is also put the original load data in the rain-flow program for obtaining the corresponding rain-flow matrix. The road load can be quantitatively analyzed after the previous processing. In order to do a brief description, it is possible to choose any side WFT and the load of any dimension. So the vertical load (Fz) on the left WFT was selected in this paper. The mean value of the Fz of the standard cobblestone road and the old cobblestone road is shown as in Fig. 10, and the amplitudes value of the Fz of the standard cobblestone road and the old cobblestone road is shown as in Fig. 11. It can be seen from Fig. 10 that the mean value of $\mathrm{Fz}$ is mainly concentrated on a quarter of the car's weight. And the effect on the standard cobblestone road and the old cobblestone road is no significant difference, which is consistent with the car weight is consistent. It is easy to see that the amplitudes value is obviously different from Fig. 11. The amplitudes value under $2000 \mathrm{~N}$ of the standard road and the old road are relatively abundant, while the number of load cycles between in $2000 \mathrm{~N}$ to $4000 \mathrm{~N}$ is not the same. The old road is clearly less than the standard one, which shows there is consumption about the old road, and it hardly provide enough loads of this level. 


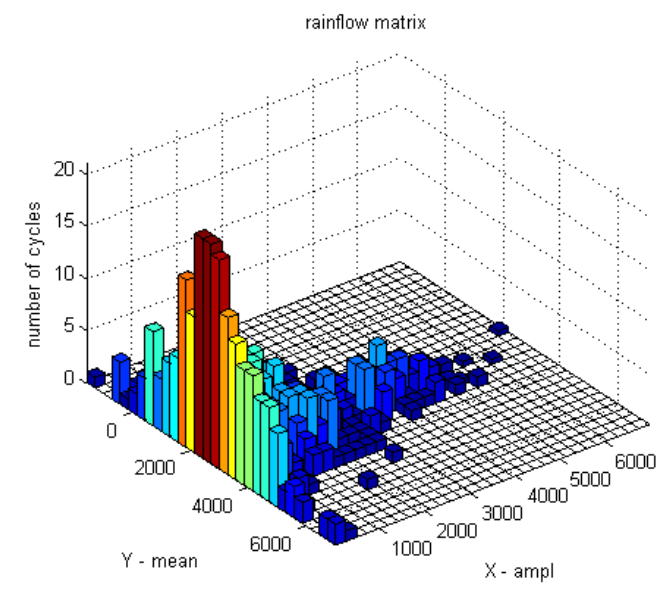

A: Rain-flow matrix of $F x$

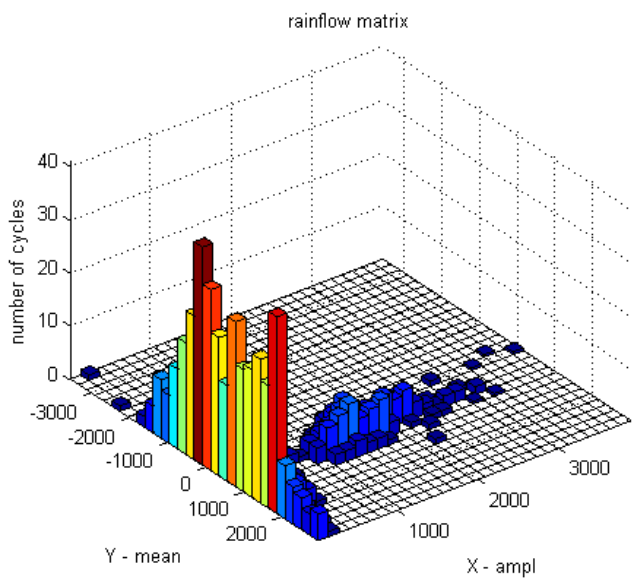

B: Rain-flow matrix of Fy

Fig. 9: Rain-flow matrix of load on the old road.

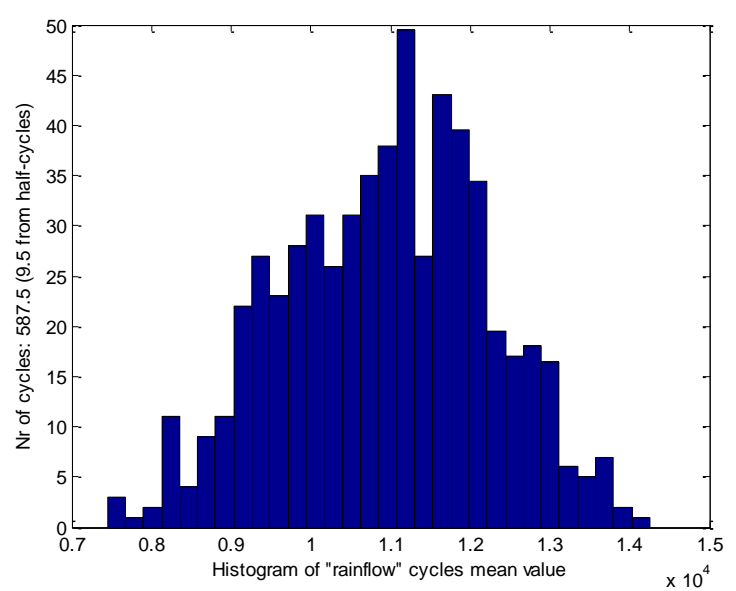

A: On the standard road.

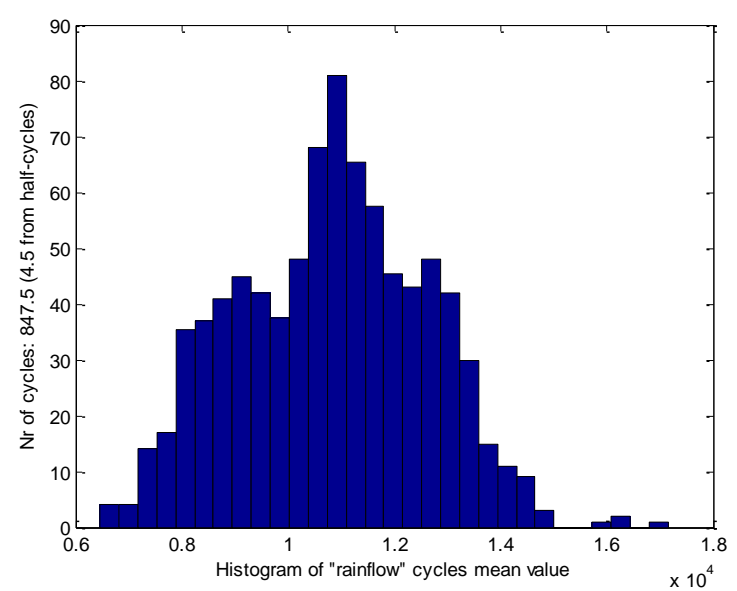

B: On the old road.

Fig. 10: The mean value of the Fz

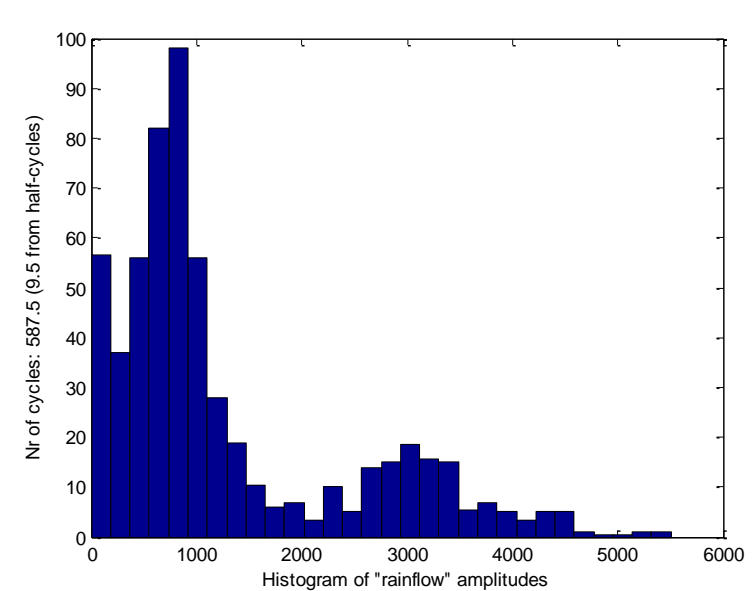

A: On the standard road.

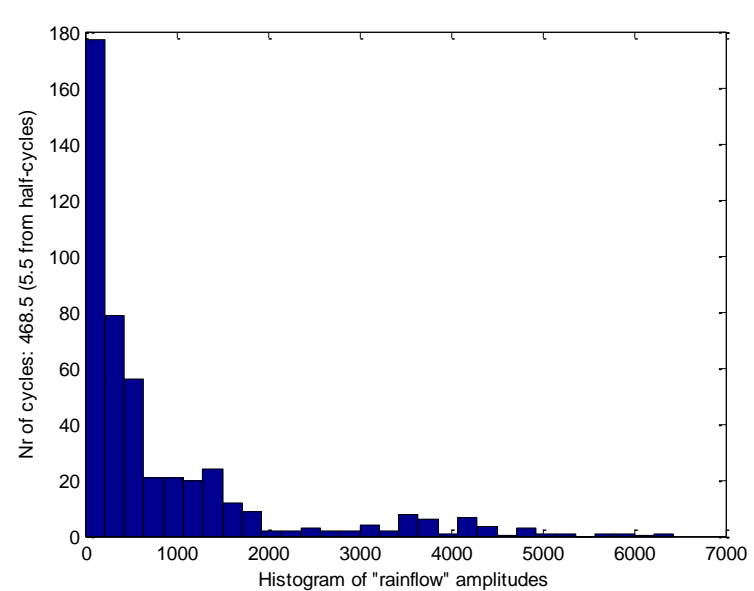

B: On the old road.

Fig. 11: The amplitudes value of the Fz

\section{Summary}

The above analysis of Fz can also be used to analyze Fx and Fy. Similarly, the left and right wheels are the same method of analysis. After completing the assessment of the road with the WFT system, the loads of the special road for each wheel can be quantified. It is clear to know how many loads the standard road or the old road can provide to the vehicle, and loads in prototype test on any road can be accurately known, which makes the prototype test easier to plan. This is a way to analyze the quality of 
the road from the perspective of the purpose of the special roads, and it reflects the nature of the special roads, so it is superior to the traditional method of using road roughness to evaluate.

\section{References}

[1]. Hong Yongfu. Automobile development engineering. Machinery Industry Press.2014, p115-160.

[2]. Duan Huming, et al. Feature parameter extraction and statistical analysis of road surface measurement data. Journal of Vibration and Shock, 2013. 32(1): p30-34.

[3]. Yong-chen, W.G, The methods of power spectrum estimation based on measured pavement. 2011, IEEE. p. 2787 - 2790.

[4]. Lin, G., et al., A self-decoupled three-axis force sensor for measuring the wheel force. Proceedings of the Institution of Mechanical Engineers, Part D: Journal of Automobile Engineering, 2014. 228(3): p. 319-334.

[5]. Hu, Y.D., et al., Study on Load Spectrum Correlation between Service Roads and Proving Ground Roads Based on Spindle Wheel Forces. Applied Mechanics and Materials, 2012. 239-240: p. $685-689$ 